\title{
INTEGRALS INVOLVING PRODUCTS OF MODIFIED BESSEL FUNCTIONS OF THE SECOND KIND
}

\author{
by F. M. RAGAB
}

(Received 1 May, 1962)

1. Introductory. It is proposed to establish the two following integrals.

$$
\begin{aligned}
& \text { If } R\left(k \pm v+\frac{1}{2} n\right)>0, \\
& \int_{0}^{\infty} \lambda^{k-1} K_{v}(\lambda) K_{\mu}\left(x \lambda^{-n}\right) d \lambda=2^{k-n-3} \pi^{-n} n^{k-1} \\
& \quad \times \sum_{i,-i} \frac{1}{i} E\left\{\Delta\left(n ; \frac{1}{2} k+\frac{1}{2} v\right), \Delta\left(n ; \frac{1}{2} k-\frac{1}{2} v\right), \frac{1}{2} \mu,-\frac{1}{2} \mu, 1:: \frac{1}{4} e^{i \pi}(2 n)^{-2 n} x^{2}\right\},
\end{aligned}
$$

where $n$ is a positive integer, $x$ is real and positive, $\mu$ and $v$ are complex, and $\Delta(n ; \alpha)$ represents the set of parameters

$$
\frac{\alpha}{n}, \frac{\alpha+1}{n}, \ldots, \frac{\alpha+n-1}{n}
$$

If $R(k \pm v \pm n \mu)>0$,

$$
\begin{aligned}
\int_{0}^{\infty} \lambda^{k-1} K_{\nu}(\lambda) K_{\mu}\left(x \lambda^{n}\right) d \lambda & =-2^{k-n-2} \pi^{2-n} n^{k-1} \sum_{\mu,-\mu} \operatorname{cosec} \mu \pi\left\{\frac{1}{4}(2 n)^{2 n} x^{2}\right\}^{\frac{1}{2} \mu} \\
& \times E\left\{\Delta\left(n ; \frac{k+n \mu+v}{2}\right) \Delta\left(n ; \frac{k+n \mu-v}{2}\right): 1+\mu: \frac{1}{4} e^{ \pm i \pi}(2 n)^{-2 n} x^{-2}\right\},
\end{aligned}
$$

where $n$ is a positive integer and $x$ is real and positive.

The following formulae will be required in the proofs.

If $p \geqq q+1$, then $[1, \mathrm{p} .409]$

$$
\begin{aligned}
& E\left(p ; \alpha_{r}: q ; \rho_{s}: z\right)=\pi^{p-q-1} \sum_{r=1}^{p} \prod_{s=1}^{q} \sin \left(\rho_{s}-\alpha_{r}\right) \pi \prod_{t=1}^{p} \operatorname{cosec}\left(\alpha_{t}-\alpha_{r}\right) \pi \\
& \times z^{\alpha_{r} E}\left\{\begin{array}{l}
\alpha_{r}, \alpha_{r}-\rho_{1}+1, \ldots, \alpha_{r}-\rho_{q}+1: \frac{e^{ \pm i \pi(p-q-1)}}{z} \\
\alpha_{r}-\alpha_{1}+1, \ldots * \ldots, \alpha_{r}-\alpha_{p}+1
\end{array}\right\} . \\
& \sin \left(\frac{k}{n} \pi\right) \sin \left(\frac{k+1}{n} \pi\right) \ldots \sin \left(\frac{k+n-1}{n} \pi\right)=2^{1-n} \sin (k \pi) \text {. } \\
& \sin \frac{\pi}{n} \sin \frac{2 \pi}{n} \ldots \sin \frac{(n-1) \pi}{n}=2^{1-n} n .
\end{aligned}
$$


If $R(k \pm v)>0, \quad[2, \mathrm{p} .119]$

$$
\begin{aligned}
& \int_{0}^{\infty} \lambda^{k-1} K_{v}(\lambda) E\left(p ; \alpha_{r}: q ; \rho_{s}: z \lambda^{-2 n}\right) d \lambda
\end{aligned}
$$

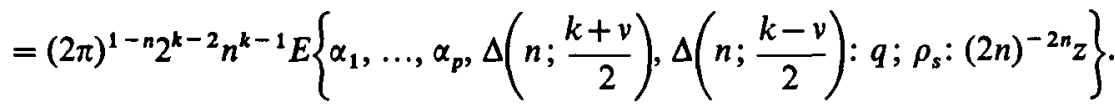

If $p \geqq q+1,[1$, p. 353$]$

$$
\begin{aligned}
E\left(p ; \alpha_{r}: q ; \rho_{s}: z\right)=\sum_{r=1}^{p} \prod_{r=1}^{p} \Gamma\left(\alpha_{t}-\alpha_{r}\right)\left\{\prod_{s=1}^{q} \Gamma\left(\rho_{s}-\alpha_{r}\right)\right\}^{-1} & \\
& \times \Gamma\left(\alpha_{r}\right) z^{\alpha_{r}} F\left\{\begin{array}{l}
\alpha_{r}, \alpha_{r}-\rho_{1}+1, \ldots, \alpha_{r}-\rho_{q}+1 ;(-1)^{p-q_{z}} \\
\alpha_{r}-\alpha_{1}+1, \ldots \ldots, \alpha_{r}-\alpha_{p}+1
\end{array}\right\} .
\end{aligned}
$$

The formula

$$
K_{\mu}(z)=\frac{1}{4 \pi} \sum_{i,-i} \frac{1}{i} E\left(1, \frac{1}{2} \mu,-\frac{1}{2} \mu:: \frac{1}{4} e^{i \pi} z^{2}\right)
$$

may be established by expanding the functions on the right by means of (7).

If $p \geqq q+1,|\operatorname{amp} z|<\pi$ and if $R\left(k \pm v+2 n \alpha_{r}\right)>0$, where $r=1,2, \ldots, p, n$ being a positive integer,

$$
\begin{aligned}
& \int_{0}^{\infty} \lambda^{k-1} K_{v}(\lambda) E\left(p ; \alpha_{r}: q ; \rho_{s}: z \lambda^{2 n}\right) d \lambda \\
& \quad=\frac{2^{k+n-3} \pi^{n+1} n^{k-1}}{\sin \left(\frac{k+v}{2} \pi\right) \sin \left(\frac{k-v}{2} \pi\right)} E\left[\begin{array}{l}
p ; \alpha_{r} \\
\Delta\left(n ; 1-\frac{k+v}{2}\right), \Delta\left(n ; 1-\frac{k-v}{2}\right), \rho_{1}, \ldots, \rho_{q}
\end{array}\right] \\
& +2^{k+n-3} \pi^{n+1} n^{k-2} \\
& \times \sum_{v,-v}\left[\begin{array}{l}
\sum_{l=0}^{n-1}(-1)^{l} \frac{\left\{(2 n)^{2 n} z\right\}^{-(k+v+2 l) / 2 n}}{\sin \left(\frac{k+v+2 l}{2 n} \pi\right) \sin (v+l) \pi} \\
\times E\left\{\begin{array}{l}
p ; \alpha_{r}+\frac{k+v+2 l}{2 n} \\
\frac{l+1}{n}, \frac{l+2}{n}, \ldots * \ldots, \frac{l+n}{n}, 1+\frac{k+v+2 l}{2 n}, \\
\Delta(n ; v+l+1), \rho_{1}+\frac{k+v+2 l}{2 n}, \ldots, \rho_{q}+-\frac{k+v+2 l}{2 n}
\end{array}\right]
\end{array}\right] .
\end{aligned}
$$


To prove this, start with the special case $p=1, q=0$; then, from (3), it follows that

$$
\int_{0}^{\infty} \lambda^{k-1} K_{v}(\lambda) E\left(\alpha:: z \hat{\lambda}^{2 n}\right) d \lambda=z^{\alpha} \int_{0}^{\infty} \lambda^{k+2 n a-1} K_{v}(\lambda) E\left(\alpha:: z^{-1} \lambda^{-2 n}\right) d \lambda
$$

and from (6) this is equal to

$$
(2 \pi)^{1-n} 2^{k+2 n \alpha-2} n^{k+2 n \alpha-1} z^{\alpha} E\left\{\alpha, \Delta\left(n ; n \alpha+\frac{k+v}{2}\right), \Delta\left(n ; n \alpha+\frac{k-v}{2}\right)::(2 n)^{-2 n} z^{-1}\right\}
$$

Here apply formula (3), using (4) and (5), and so obtain (9) with $p=1, q=0$. Formula $(9)$ is then obtained by generalising.

If $n$ is a positive integer

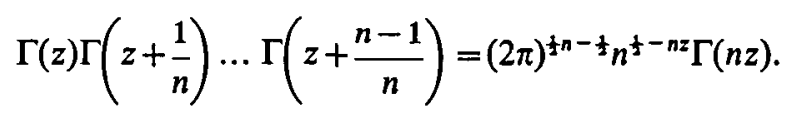

If $p \leqq q$, then $[1, \mathrm{p} .352]$

$$
E\left(p ; \alpha_{r}: q ; \rho_{s}: z\right)=\frac{\Gamma\left(\alpha_{1}\right) \ldots \Gamma\left(\alpha_{p}\right)}{\Gamma\left(\rho_{1}\right) \ldots \Gamma\left(\rho_{q}\right)} F\left(\begin{array}{c}
\alpha_{1}, \ldots, \alpha_{p} ; z \\
\rho_{1}, \ldots, \rho_{q}
\end{array}\right)
$$

2. Proofs of the integrals. To prove (1) apply (6) twice with $p=3, q=0, \alpha_{1}=1, \alpha_{2}=\frac{1}{2} \mu$ and $\alpha_{3}=-\frac{1}{2} \mu$. In the first case take $z=\frac{1}{4} x^{2} e^{i \pi}$, in the second take $z=\frac{1}{4} x^{2} e^{-i \pi}$, divide by $i$ and subtract, and then apply (8).

In order to obtain (2) take $p=3, q=0$ in (9) with $\alpha_{1}=\frac{1}{2} \mu, \alpha_{2}=-\frac{1}{2} \mu, \alpha_{3}=1$; then combine the two cases in which $z=\frac{1}{4} x^{2} e^{i \pi}$ and $z=\frac{1}{4} x^{2} e^{-i \pi}$, using (8). As the terms arising from the first $E$-function on the right of (9) cancel, it follows that

$$
\begin{aligned}
& \int_{0}^{\infty} \lambda^{k-1} K_{v}(\lambda) K_{\mu}\left(x \lambda^{n}\right) d \lambda=-2^{k+n-4} \pi^{n} n^{k-2} \\
& \quad \times \sum_{v_{v}-v}\left[\sum_{=0}^{n-1} \frac{\left\{(2 n)^{2 n} x^{2} / 4\right\}^{-(k+v+2 l) / 2 n}}{\sin v \pi} E\left\{\begin{array}{l}
\frac{\mu}{2}+\frac{k+v+2 l}{2 n},-\frac{\mu}{2}+\frac{k+v+2 l}{2 n}:-(2 n)^{2 n} x^{2} / 4 \\
\frac{l+1,}{n} \ldots * \ldots, \frac{l+n}{n}, \Delta(n ; v+l+1)
\end{array}\right\}\right] .
\end{aligned}
$$


Here apply (11) and (10), and the expression becomes $-2^{k-3} \pi n^{k+v-1}$

$$
\times \sum_{v,-v}\left[\begin{array}{c}
\sum_{i=0}^{n-1} \frac{\left\{(2 n)^{2 n} x^{2} / 4\right\}^{-(k+v+2 l) / 2 n} n^{2 l} \Gamma\left(\frac{\mu}{2}+\frac{k+v+2 l}{2 n}\right) \Gamma\left(-\frac{\mu}{2}+\frac{k+v+2 l}{2 n}\right)}{\sin v \pi \Gamma(l+1) \Gamma(v+l+1)} \\
\times F\left(\begin{array}{l}
\left.\frac{\mu}{2}+\frac{k+v+2 l}{2 n},-\frac{\mu}{2}+\frac{k+v+2 l}{2 n} ; \frac{4}{(2 n)^{2 n} x^{2}}\right) \\
\frac{l+1}{n}, \ldots * \ldots, \frac{l+n}{n}, \Delta(n ; v+l+1)
\end{array}\right.
\end{array}\right] .
$$

Again, apply (7) to the expression on the right of (2), so getting

$-2^{k-n-2} \pi^{2-n} n^{k-1} \sum_{\mu_{1},-\mu} \operatorname{cosec} \mu \pi\left\{\frac{1}{4}(2 n)^{2 n} x^{2}\right\}^{\frac{1}{2 \mu}}$

$\times \sum_{v_{0}-v} \sum_{l=0}^{n-1} \frac{\Gamma\left(-\frac{l}{n}\right) \ldots \Gamma\left(-\frac{1}{n}\right) \Gamma\left(\frac{1}{n}\right) \ldots \Gamma\left(\frac{n-l-1}{n}\right) \Gamma\left(\frac{-v-l}{n}\right) \ldots \Gamma\left(\frac{-v-l+n-1}{n}\right)}{\Gamma\left(1+\mu-\frac{\mu n+k+v+2 l}{2 n}\right)}$

$\times \Gamma\left(\frac{\mu n+k+\nu+2 l}{2 n}\right)\left\{\frac{4 e^{ \pm i n}}{(2 n)^{2 n} x^{2}}\right\}^{(\mu n+k+v+2 l) / 2 n}$

$\times F\left\{\begin{array}{lr}\frac{\mu n+k+v+2 l}{2 n}, \frac{-\mu n+k+v+2 l}{2 n} & : 4(2 n)^{-2 n} x^{-2} \\ 1+\frac{l}{n}, 1+\frac{l-1}{n}, \ldots, 1+\frac{1}{n}, 1-\frac{1}{n}, \ldots, 1-\frac{n-l-1}{n}, \Delta(n ; v+l+1)\end{array}\right\}$.

Here replace

$$
1 / \Gamma\left(1+\mu-\frac{\mu n+k+v+2 l}{2 n}\right)
$$

by

$$
\Gamma\left(\frac{-\mu n+k+v+2 l}{2 n}\right) \sin \left(\frac{-\mu n+k+v+2 l}{2 n} \pi\right) / \pi
$$

Then note that

$$
\sum_{\mu_{0}=-\mu} \sin \left(\frac{-\mu n+k+\nu+2 l}{2 n} \pi\right) e^{ \pm i \pi(\mu n+k+v+2 l) / 2} / \sin \mu \pi=-1 .
$$


Also, from (10) it follows that

$$
\Gamma\left(\frac{-v-l}{n}\right) \ldots \Gamma\left(\frac{-v-l+n-1}{n}\right)=(-1)^{1-1} \frac{(2 \pi)^{\frac{1}{2 n-\frac{1}{2}}} n^{\frac{1}{2}+v+l} \pi}{\Gamma(v+l+1) \sin v \pi},
$$

and that

$$
\Gamma\left(-\frac{l}{n}\right) \ldots \Gamma\left(-\frac{1}{n}\right) \Gamma\left(\frac{1}{n}\right) \ldots \Gamma\left(\frac{n-l-1}{n}\right)=(-1)^{l} \frac{(2 \pi)^{\frac{1}{2} n-\frac{1}{2}} n^{l-\frac{1}{2}}}{\Gamma(l+1)}
$$

Hence the expression (B) is equal to the expression (A), so that formula (2) has been established.

\section{REFERENCES}

1. T. M. MacRobert, Functions of a complex variable, 5th edn. (London, 1962).

2. F. M. Ragab, An integral involving a modified Bessel function of the second kind and an E-function, Proc. Glasgow Math. Assoc. 1 (1953), 119-120.

FACUlTy OF ScIENCE

CAIRo UNIVERSITY

CAIRO, U.A.R. 